\title{
Venetoclax-based chemotherapy in acute and chronic myeloid neoplasms: literature survey and practice points
}

\author{
Naseema Gangat [1] and Ayalew Tefferi ${ }^{1}$
}

\begin{abstract}
Venetoclax (VEN), a small-molecule inhibitor of B cell leukemia/lymphoma-2, is now FDA approved (November 2018) for use in acute myeloid leukemia (AML), specific to newly diagnosed elderly or unfit patients, in combination with a hypomethylating agent (HMA; including azacitidine or decitabine) or low-dose cytarabine. A recent phase-3 study compared VEN combined with either azacitidine or placebo, in the aforementioned study population; the complete remission (CR) and CR with incomplete count recovery (CRi) rates were $28.3 \%$ and $66.4 \%$, respectively, and an improvement in overall survival was also demonstrated. VEN-based chemotherapy has also shown activity in relapsed/ refractory AML (CR/CRi rates of 33-46\%), high-risk myelodysplastic syndromes (CR 39\% in treatment naïve, 5-14\% in HMA failure), and blast-phase myeloproliferative neoplasm (CR 25\%); in all instances, an additional fraction of patients met less stringent criteria for overall response. Regardless, venetoclax-induced remissions were often short-lived (less than a year) but long enough to allow some patients transition to allogeneic stem cell transplant. Herein, we review the current literature on the use of VEN-based combination therapy in both acute and chronic myeloid malignancies and also provide an outline of procedures we follow at our institution for drug administration, monitoring of adverse events and dose adjustments.
\end{abstract}

\section{Introduction}

Venetoclax (ABT-199) is a BH3-mimetic agent, a highly selective inhibitor of the anti-apoptotic protein $\mathrm{B}$ cell leukemia/lymphoma (BCL-2), which was discovered in 1984 as part of translocation $\mathrm{t}(14 ; 18)$ in follicular lymphoma $^{1-3}$. Since then several anti-apoptotic: BCLX ${ }_{\mathrm{L}}$, MCL1, BCL-W and pro-apoptotic proteins: BAX, BAK, and $\mathrm{BOK}$, and the $\mathrm{BH} 3$-only proteins $\mathrm{BIM}, \mathrm{BAD}, \mathrm{BID}$, BIK, NOXA, and PUMA have been identified. Overexpression of $\mathrm{BCL}-2, \mathrm{BCLX}_{\mathrm{L}}$, and MCL1 frequently occurs in acute myeloid leukemia (AML) conferring resistance to conventional chemotherapy ${ }^{4}$. Initial clinical efforts with targeting anti-apoptotic proteins centered on navitoclax (ABT-263), a BH3 mimetic that binds to BCL$2, \mathrm{BCL}-\mathrm{XL}$, and $\mathrm{BCL}-\mathrm{W}^{5}$. As megakaryocytes are BCL-XL dependent, navitoclax caused significant dose-limiting

Correspondence: Naseema Gangat (gangat.naseema@mayo.edu)

${ }^{1}$ Division of Hematology, Mayo Clinic, Rochester, MN, USA thrombocytopenia limiting its utility in AML and other myeloid malignancies ${ }^{6}$. In additional preclinical work with ABT-737, an agent with similar activity to navitoclax, successful elimination of blasts in AML cell lines and patient samples was accompanied by eradication of BCL2-dependent leukemia stem cells while sparing normal hematopoietic stem cells which rely on MCL1 for survi$\mathrm{val}^{7}$. On the other hand, venetoclax, which is a modified BH3-mimetic derivative of navitoclax, maintains specificity for BCL-2 but lacks affinity for BCL-XL. Venitoclax also exhibited potent anti-leukemic activity in AML cell lines, patient samples, and xenograft murine models ${ }^{8}$. Interestingly, AML cell lines with MLL-fusion and samples from acute promyelocytic leukemia patients were particularly sensitive to venetoclax therapy ${ }^{9}$. Moreover, in preclinical models, synergy with the hypomethylating agent (HMA) azacitidine, which inhibits MCL1, was also established $^{10,11}$. Together, these findings suggested promising activity of venetoclax in AML and laid the 
groundwork for clinical studies. In the current review, we summarize retrospective observations and clinical trials with venetoclax-based regimens in AML, including those that led to its FDA approval in November 2018 for treatment naïve elderly or unfit AML. Additionally, we share preliminary observations from ongoing studies in myelodysplastic syndromes (MDS) and related chronic myeloid malignancies. Furthermore, we expand upon practice relevant issues that are frequently encountered with use of venetoclax-based chemotherapy.

\section{Venetoclax as upfront therapy in AML}

Venetoclax in combination with HMA or low-dose cytarabine has undergone extensive clinical evaluation in AML, with Phase 1b/II clinical trial data forming the basis of its FDA approval in November 2018 for treatment naïve, elderly $>75$ years old or unfit AML patients ${ }^{12,13}$. Please refer to Table 1 for a comprehensive summary of results from all published clinical trials and retrospective studies with venetoclax-based chemotherapy in treatment naïve AML. The initial Phase $1 \mathrm{~b}$ study with venetoclax plus HMA in untreated AML comprised of three patient cohorts, namely venetoclax plus either (i) decitabine (Group A), (ii) azacitidine (Group B), or (iii) decitabine in addition to posaconazole (Group C), which established the recommended phase 2 dose of venetoclax at 400 or $800 \mathrm{mg}$ daily $^{12}$. In terms of responses, overall response rates were $62 \%$ with complete remission (CR) achieved in $27 \%$ with venetoclax plus azacitidine or $35 \%$ with decitabine $^{12}$. In a subsequent phase $1 \mathrm{~b} / \mathrm{II}$ trial in AML patients above 65 years of age that were unfit for intensive chemotherapy, administration of venetoclax 400 or $800 \mathrm{mg}$ with either azacitidine or decitabine resulted in a composite response (complete remission (CR, 37\%)/CR with incomplete hematological recovery (CRi; 30\%) rate of $67 \%)^{13}$. Notably, a rapid median time to first response at 1.2 months, with a median duration of response of 11.3 months, and superior median overall survival of 17.5 months were observed. Moreover, responses held across the spectrum of unfavorable cytogenetic and molecular genetic abnormalities such as TP53, FLT3, IDH1/2 mutations. As expected, common adverse events included febrile neutropenia in $43 \%$ followed by myelosuppression in a quarter, and mild-moderate gastrointestinal toxicity. Early 30 -day mortality was low $(<5 \%)$. It should be emphasized that the study cohort comprised of a substantial proportion of patients harboring adverse cytogenetic anomalies (50\%), with a quarter of patients with TP53 or IDH1/2 mutations and secondary AML. In order to elucidate the mechanism underlying response to venetoclax-based therapy, Pollyea et al. ${ }^{14}$ studied 33 patients treated with azacitidine plus venetoclax, and discovered treatment-induced disturbances in the tricarboxylic acid cycle with reduction in alpha ketoglutarate, increase in succinate levels along with inhibition of the electron transport chain, thereby eradicating leukemia stem cells ${ }^{14}$. The recently published VIALE-A phase- 3 randomized study included elderly $>75$ years or younger AML patients if comorbidities precluded intensive therapy but was exclusive of patients with favorable cytogenetics $(t(8 ; 21$, inv $16, t(15 ; 17))$, prior HMA exposure, or AML arising from myeloproliferative neoplasms (MPN). The study not only confirmed a superior response rate of $66 \%$ with combination of azactidine plus venetoclax vs $28 \%$ with azacitidine alone but also demonstrated an overall survival advantage of 5 months for venetoclax with azacitidine with a median overall survival of 14.7 months vs 9.6 months with azacitidine alone $\mathrm{e}^{15}$. Moreover, superior responses occurred in NPM1 (67\% vs $24 \%$ ), FLT3 (72\% vs $36 \%$ ), IDH1/2 (75\% vs $11 \%$ ), and TP53 (55\% vs $0 \%)$ mutated patients when treated with a combination of azacitidine and venetoclax as opposed to azacitidine alone ${ }^{15}$.

Dinardo et al. ${ }^{16}$ shared phase 2 results with a 10-day course of decitabine $20 \mathrm{mg} / \mathrm{m}^{2}$ with venetoclax $400 \mathrm{mg}$ daily for induction followed by 5 days of decitabine with venetoclax in consolidation in 70 treatment-naïve elderly AML patients over 60 years that were ineligible for intensive therapy. Unsurprisingly, decitabine dose reductions were instituted in $13 \%$ with over $90 \%$ of patients receiving 21 days or less of venetoclax with infectious complications recorded in half of patients. CR/CRi was achieved in $84 \%$, with minimal residual disease (MRD) negativity in $67 \%$, with high responses across all ELN-risk groups; CR/CRi rates of $90 \%, 100 \%$, and $75 \%$ among favorable, intermediate, and adverse risk groups, respectively $^{16}$. Similarly, CR/CRi rates among NPM1, IDH1/2, N/Kras, and TP53-mutated patients were $95 \%, 84 \%, 74 \%$, and $69 \%$, respectively. Importantly, responses were less durable and similar with a 5- vs 10-day course of decitabine plus venetoclax in TP53 mutated patients. In contrast, for all treatment-naïve patients median duration of response was not reached, with a median overall survival of 18.1 months $^{16}$. Interestingly, among 14 newly diagnosed FLT3-mutated patients, ten received FLT3 inhibitors in addition to decitabine and venetoclax, achieving CR/CRi rates and MRD negativity by PCR in $86 \%$ with three patients transitioning to allogeneic hematopoietic stem cell transplant (AHSCT) ${ }^{16}$.

Venetoclax is also administered in combination with low-dose cytarabine in AML, particularly in patients experiencing disease progression on HMA therapy ${ }^{17,18}$. This regimen was studied in a phase 1b/II trial in which venetoclax $600 \mathrm{mg}$ orally daily was administered in combination with low-dose cytarabine $20 \mathrm{mg} / \mathrm{m}^{2}$ subcutaneously days 1-10 to elderly AML patients, half with secondary AML, and one-third each with poor-risk cytogenetics or prior HMA exposure ${ }^{17}$. Among 82 


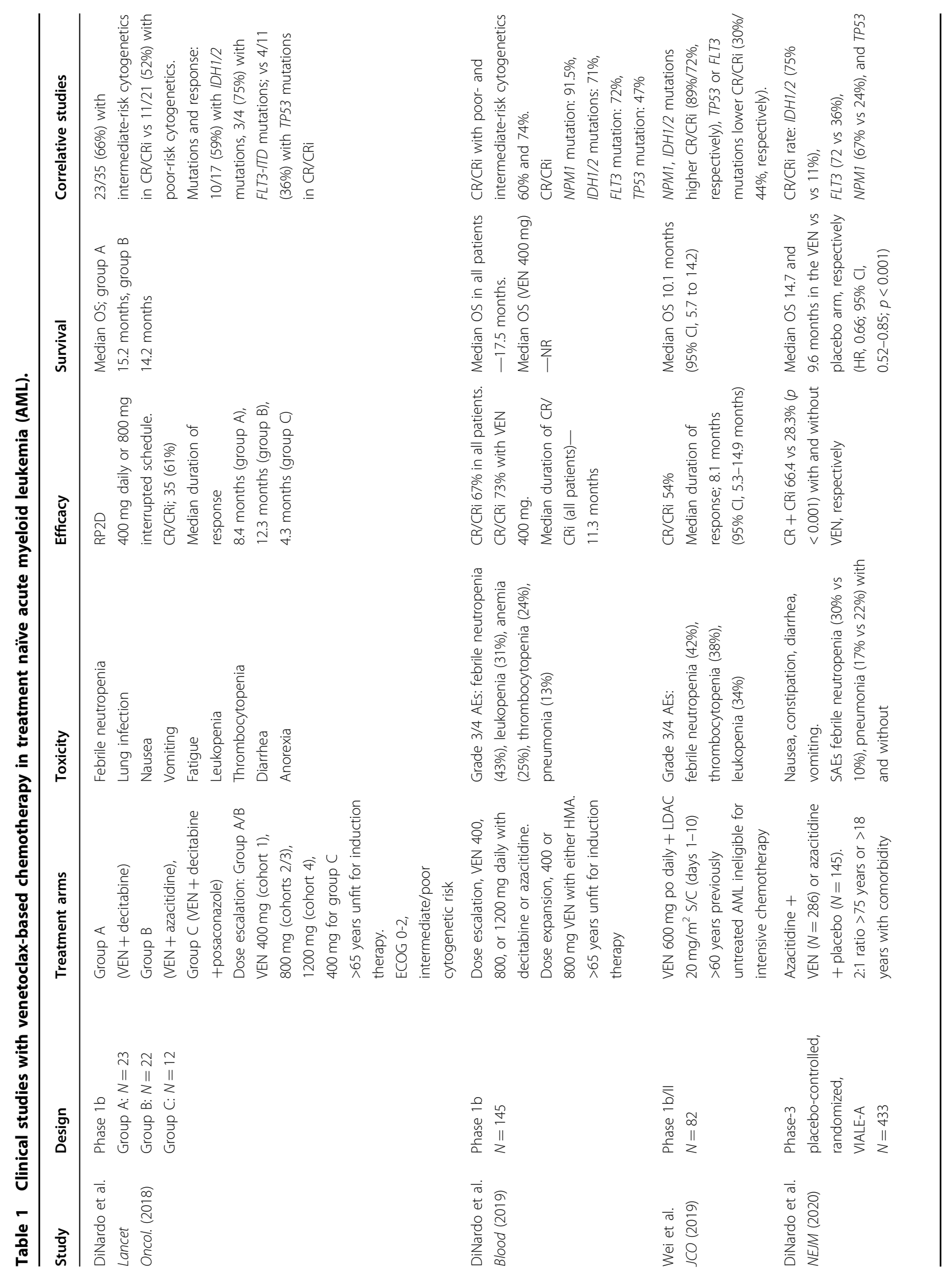




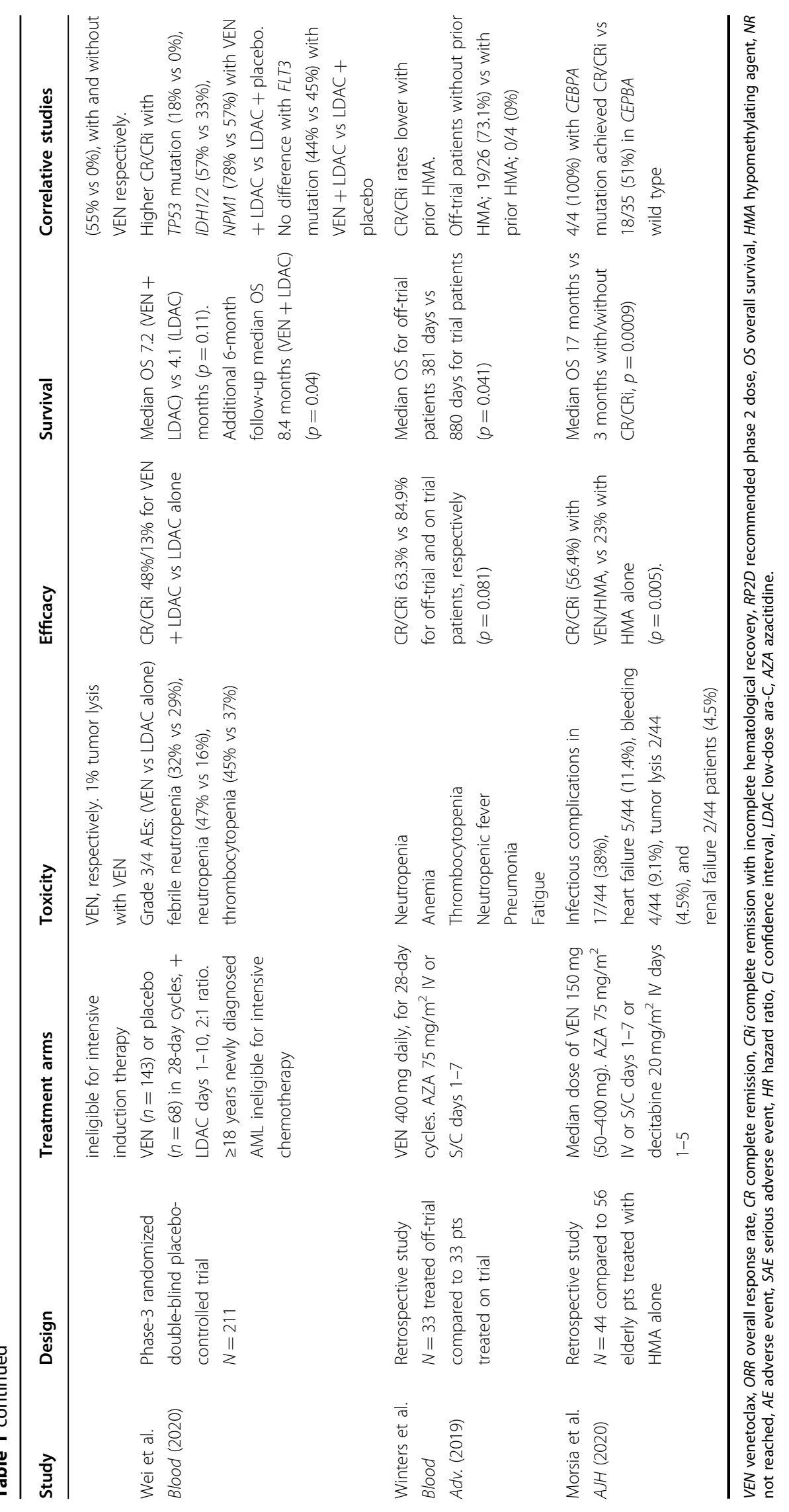


elderly AML patients (median age: 74 years) treated on study, CR/CRi rates were $54 \%$ with CR rate of $21 \%$. As expected, higher responses were noted with de novo AML, intermediate-risk cytogenetics, and in the absence of prior HMA exposure. Once again, median time to CR/ CRi was rapid at 1.4 months with median duration of response of 8 months. Median overall survival for the entire cohort was 10.1 months, with distinct survival differences appreciated based on prior HMA use (4.1 vs 13.5 months, respectively, with or without prior HMA). The follow-up randomized phase-3 study, VIALE-C which included AML patients $>18$ years of age ineligible for intensive therapy, also confirmed superior CR/CRi rate at $48 \%$ with venetoclax plus low-dose cytarabine compared to $13 \%$ with cytarabine alone ${ }^{18}$. Upon initial survival analysis, an overall survival benefit with the combination of venetoclax and low-dose cytarabine was not apparent; however, with an additional 6-month follow-up, survival distinctions emerged with a median overall survival of 8.4 months for venetoclax plus cytarabine vs 4.1 months with cytarabine alone. Superior responses were recorded with combination therapy among NPM1 (78\% vs $57 \%$ ), IDH1/2 (57\% vs 33\%), and TP53 (18\% vs $0 \%)$ mutated patients with no difference noted in FLT3-mutated patients ( $44 \%$ vs $45 \%)$.

Moving beyond clinical trials which are fraught with issues of selection bias and vigilant monitoring, Winters et al. shared their real-world experience with azacitidine plus venetoclax in AML. Thirty-three patients treated with azacitidine plus venetoclax off-trial $(n=33)$ at their institution were compared with trial patients treated with the same regimen. Not surprisingly, lower response rates ( $63 \%$ vs $85 \%, p=0.08)$ with consequently shortened survival (381 vs 880 days, $p=0.04$ ) were noted among offtrial patients ${ }^{19}$. Moreover, none of four patients with prior HMA exposure responded with 19 of 26 (73.1\%) patients without prior HMA responding to therapy. On that note, we have recently published our Mayo clinic experience with off-trial use of HMA plus venetoclax among 44 treatment-naïve AML patients of median age 73.5 years, which were enriched with secondary, therapy related and ELN adverse risk disease. We found encouraging responses with $\mathrm{CR} / \mathrm{CRi}$ rate of $50 \%$, albeit lower than that of clinical trial reports ${ }^{20}$. Remarkably, one-third of our patients achieved response after three or fewer cycles of therapy and four patients (9.1\%) proceeded to AHSCT. Prior HMA exposure did not impact response outcome with three of five such patients achieving CR/CRi. Another noteworthy observation from our study was the association of CEPBA biallelic mutations with a favorable response, with all four patients harboring $C E P B A$ biallelic mutations responding to therapy vs 18 of 35 (51\%) CEBPA wild-type patients. Even though CR/CRi rates in our series were superior with HMA plus venetoclax in comparison to a historical cohort of elderly AML patients treated with HMA alone (50\% vs $23 \%$ ), a substantial improvement in median overall survival was not detected; median overall survival of 11 months with HMA plus venetoclax vs 9.5 months with HMA alone ${ }^{20,21}$.

\section{Venetoclax as salvage therapy in AML}

In the foremost Phase II study of venetoclax in AML, the drug was administered as monotherapy at $800 \mathrm{mg}$ daily to 30 patients with relapsed/refractory disease, exhibiting fairly limited activity with an overall response rate of $19 \%^{22}$. However, it was striking that one-third of patients in $\mathrm{CR} / \mathrm{CRi}$, harbored IDH1/2 mutations, consistent with later reports of venetoclax combination therapy in treatment-naïve AML reaffirming the sensitivity of $I D H 1 / 2$ mutated patients to venetoclax-based therapy $^{22-24}$. A recent phase II study with a 10-day induction course of decitabine along with venetoclax in 55 relapsed/refractory AML patients of which one-third were relapsed post AHSCT showed reasonable efficacy ${ }^{16}$. Remarkably, CR/CRi rates were $42 \%$ with half of patients achieving MRD negativity with favorable responses in NPM1, IDH1/2, and FLT3 mutated patients ${ }^{16}$. Moreover, median duration of response was 16.8 months with median overall survival of 7.8 months. Among 12 patients with previously treated FLT3 mutated AML, 8 with prior FLT3 inhibitor exposure, all received FLT3 inhibitors along with decitabine and venetoclax achieving a CR/CRi rate of $42 \%$, with MRD negativity by flow cytometry and PCR in half and quarter of responding patients respectively. Furthermore, four patients proceeded to AHSCT ${ }^{16}$.

Since approval of venetoclax in 2018 for upfront use in AML, it has gained popularity as a salvage regimen with a handful of published retrospective reports that are delineated in Table 2 (refs. ${ }^{20,25-34}$ ). We recommend exercising caution while interpreting findings from these reports due to immense heterogeneity in patient population studied (inclusion of relapsed MDS, other myeloid malignancies, prior HMA exposure, post AHSCT), in addition to variations in dose and schedule of treatment regimens utilized either as monotherapy, combination with HMA or low-dose cytarabine. As a result, reported responses with venetoclax-based regimens in the relapsed AML setting are highly variable. For instance in an MD Anderson series $(n=43)$ which also included two patients each with MDS and blastic plasmacytoid dendritic cell neoplasm, 72\% received HMA and the remainder lowdose cytarabine, with overall response rate of $21 \%$ comprising of two patients in $\mathrm{CR}$, three in $\mathrm{CRi}$, and four in morphological leukemia free state (MLFS) ${ }^{25}$. In contrast, a study from City of Hope $(n=33)$, in which relapsed AML patients were treated with venetoclax in conjunction with HMA (decitabine, 5-day or 10- day course $(n=15$ and 16, respectively), azacitidine $(n=2)$, an overall response rate 


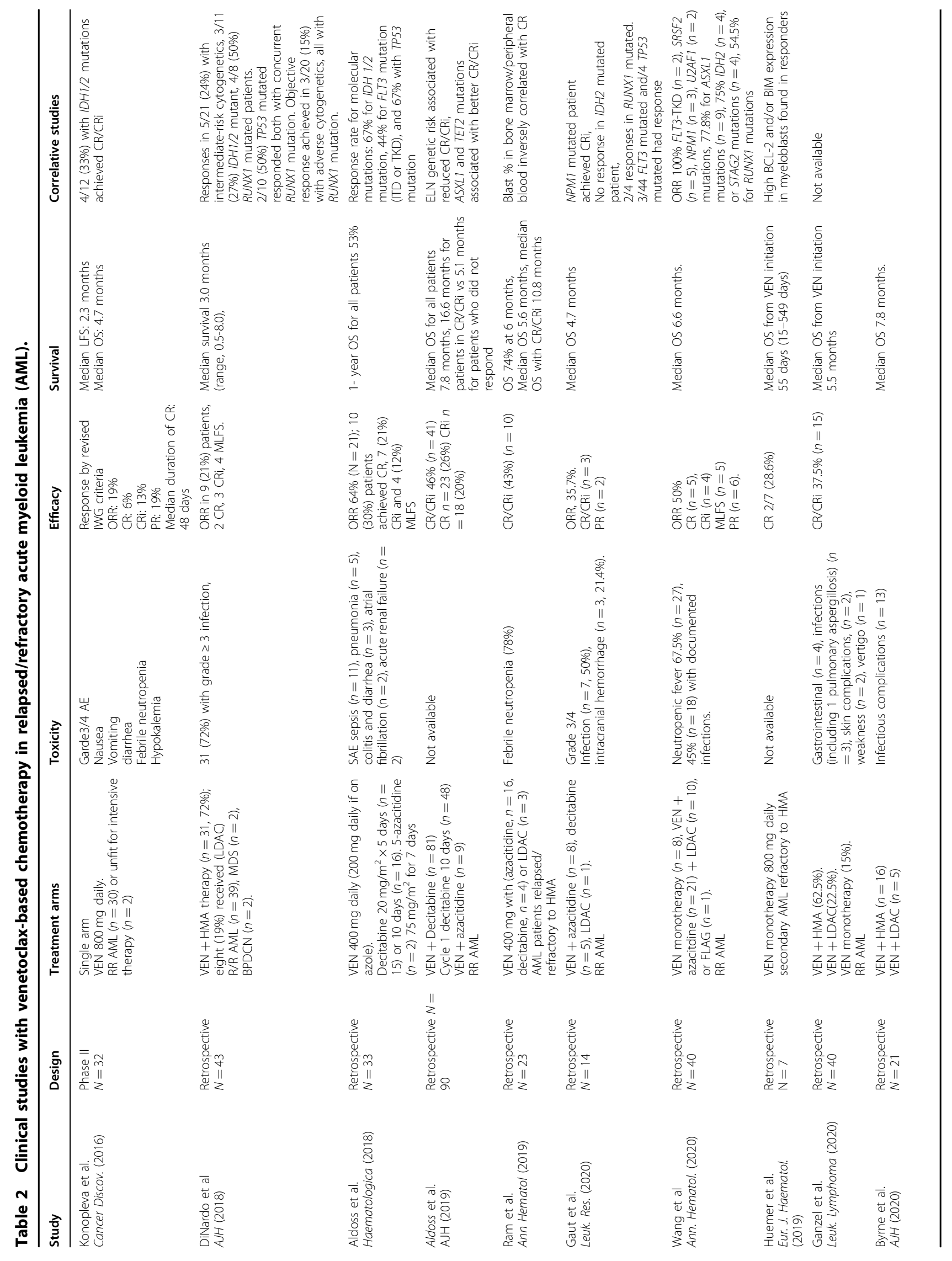




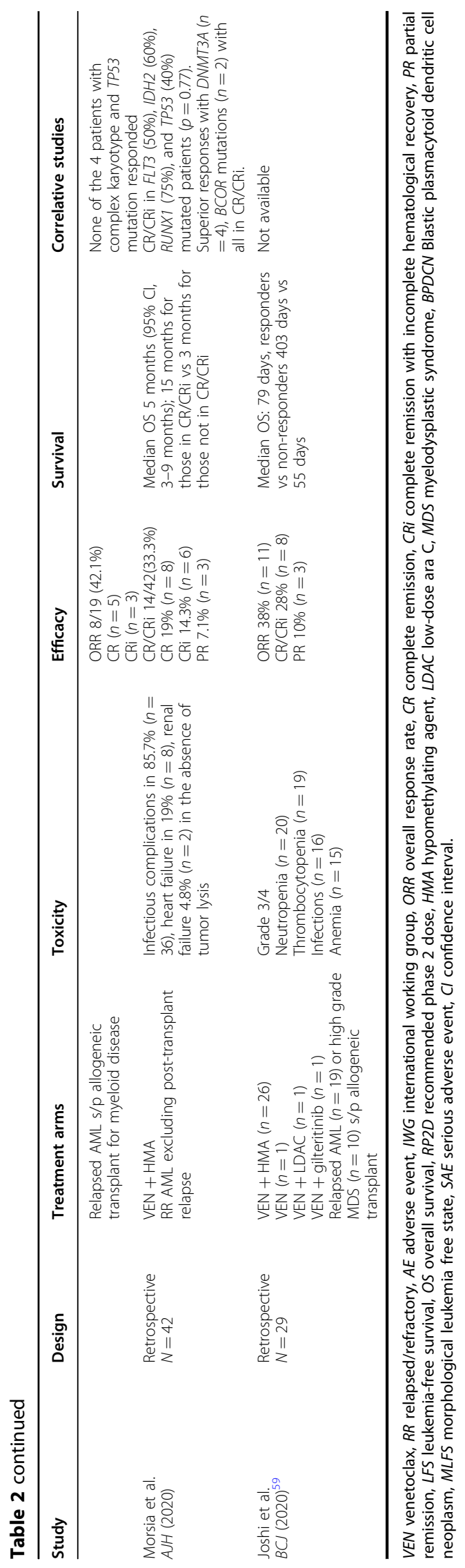

of $64 \%$ with ten patients in CR, seven in CRi, and four in MLFS were reported ${ }^{26}$. These two studies also differed in regard to predictors of response with high response rate of $50 \%$ in RUNX1-mutated patients in the MD Anderson series $^{25}$; on the other hand $44 \%$ of FLT3-mutated patients responded in the latter study ${ }^{26}$. However, both studies demonstrated similar responses among TP53-mutated patients of $50 \%$ and $67 \%$, respectively. A follow-up updated analysis of 90 relapsed AML patients treated at City of Hope, half with prior HMA use, and a third relapsed post-transplant yielded CR/CRi rates of $46 \%$, with TET2 and ASXL1 mutations associated with an improved response ${ }^{34}$. Moreover, one-third of responders from this study proceeded to AHSCT ${ }^{34}$.

In our Mayo clinic experience with venetoclax plus HMA as salvage therapy in relapsed AML exclusive of post-transplant relapse $(n=42)$, we observed CR/CRi rates of $33 \%$, with similar responses across the mutational spectrum; FLT3 (50\%), IDH2 (60\%), RUNX1 (75\%), and TP53 (40\%) mutated patients. Furthermore, 8 of 42 patients (19.1\%) were successfully bridged to $\mathrm{AHSCT}^{20}$. Similarly, a recent meta-analysis which included 224 patients with relapsed AML treated with venetoclax monotherapy or combination therapies demonstrated an overall response rate of $34.7 \%{ }^{35}$.

In regard to venetoclax use as salvage therapy in post AHSCT patients, a recent review of 21 patients solely focused on relapsed AML following transplant performed for either AML $(n=16)$ or chronic myeloid malignancy $(n=2$, MDS, $n=1$ each with chronic myelomonocytic leukemia (CMML) and primary myelofibrosis) ${ }^{32}$. The majority of patients received venetoclax with HMA $(n=16)$. Eight of 19 evaluable patients (42\%) responded with CR $(n=5)$ and CRi $(n=3)$. It is to be noted that half of these patients had relapsed within 6 months of transplant with a quarter within 100 days attesting to a population with dismal outcomes, despite which responses were sustained beyond 3 months in most patients with only one patient in CR/CRi progressing after 9 months of therapy. Moreover, four of eight patients were subsequently salvaged with either donor lymphocyte infusion $(n=2)$ or a second transplant $(n=2)$. Contrary to favorable responses reported in TP53 mutated patients ${ }^{36}$, all four patients with complex karyotype and TP53 mutation did not respond to therapy.

\section{Venetoclax in MDS and other chronic myeloid malignancies}

Venetoclax is being investigated in clinical trials in both treatment naïve high-risk MDS and MDS with progression on HMA. Table 3 provides early observations from ongoing studies in MDS. A phase 1b study in treatment naïve MDS includes patients with intermediate 2 or high International Prognostic Scoring System (IPSS) risk 

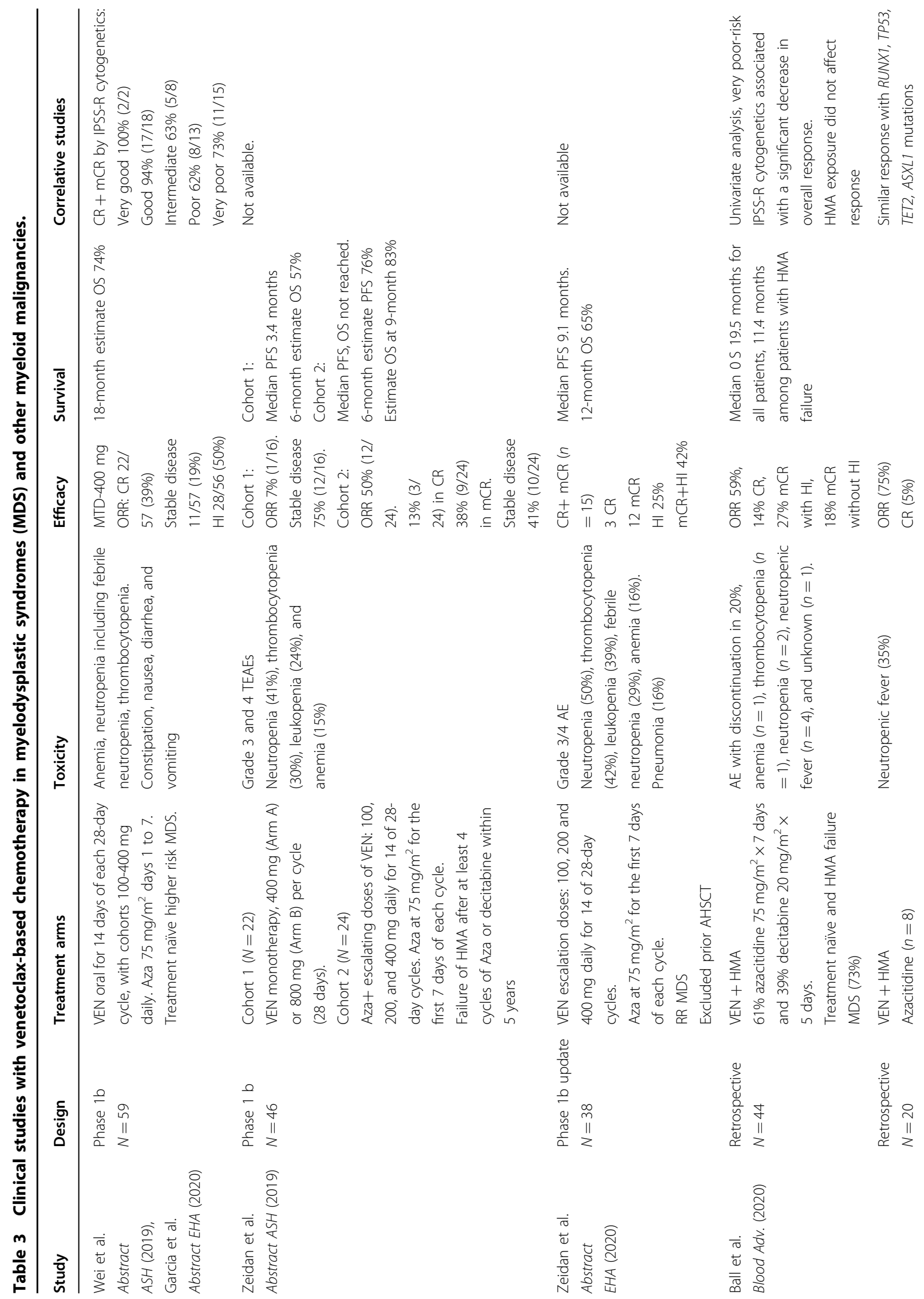


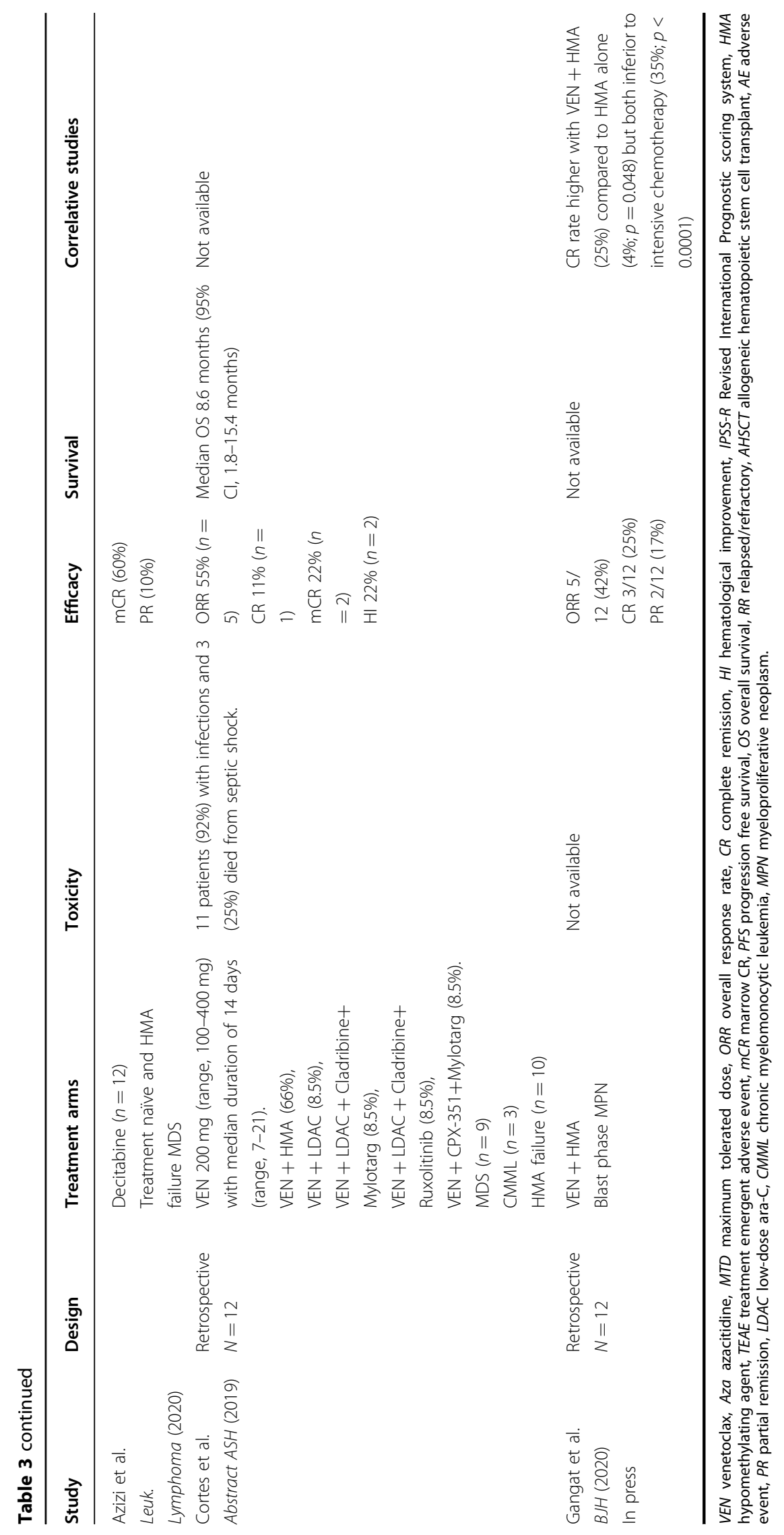


categories or intermediate, high or very high Revised IPSS (IPSS-R) categories with $<20 \%$ bone marrow blasts ${ }^{37}$. Of note, the above study excluded therapy-related MDS, myelodysplastic/myeloproliferative (MDS/MPN) overlap entities, and prior chemotherapy or AHSCT. Updated results were presented at the American society of hematology (ASH) annual meeting in 2019; a total of 59 treatment naïve MDS patients were treated with azacitidine and venetoclax on the dose escalation phase $(100$, 200, $400 \mathrm{mg}$ venetoclax daily, $n=25$ ) establishing $400 \mathrm{mg}$ daily as the recommended dose for 14 days of a 28-day cycle followed by an expansion cohort $(n=22)$. Unlike AML, no dose ramp up was performed. Common toxicities consisted of myelosuppression and gastrointestinal symptoms, with febrile neutropenia in one-third of patients. Of the 57 patients evaluated for response, median time to response was 1 month (range: $0.7-3.5$ months) with 18 patients (32\%) achieving CR, 22 patients (39\%) in marrow CR (mCR), and hematological improvement in 28 patients. Despite short follow-up, ten patients proceeded to AHSCT and an 18-month survival estimate for the cohort was $74 \%$. Furthermore, when responses were correlated with IPSS-R cytogenetic categories, $\mathrm{CR} / \mathrm{mCR}$ was achieved in almost all patients in the very good $(100 \%)$ and good (94\%) risk groups vs $62 \%$ and $73 \%$ in the poor and very poor-risk categories, respectively ${ }^{38}$.

Venetoclax-based therapy is also under investigation in MDS with disease progression after four cycles of HMA, administered as monotherapy in 22 patients $(400 \mathrm{mg}$ vs $800 \mathrm{mg}$ per 28-day cycle) and in conjunction with azacitidine with escalating doses of venetoclax $(100,200$, and $400 \mathrm{mg}$ for 14 days) in 24 patients $^{39}$. With monotherapy, overall responses were low occurring in only one of sixteen patients with stable disease in the majority ( 12 of 16 patients). Improved overall responses were recorded in half of patients treated with combination therapy, with $13 \%$ and $38 \%$ achieving $\mathrm{CR}$, and $\mathrm{mCR}$, respectively. Moreover four patients proceeded to AHSCT. Updated results of 37 evaluable patients with relapsed MDS excluding CMML and post AHSCT, treatment with combination therapy resulted in CR $(n=3)$ plus $\mathrm{mCR}$ $(n=12)$ in a total of 15 patients $(40 \%)$ with median time to response of 1.2 months. An additional $25 \%$ of patients experienced hematological improvement with red cell or platelet transfusion independence noted in one-third of patients. An encouraging median progression free survival of 9 months with a 1 year overall survival estimate of $65 \%$ was also reported ${ }^{40}$.

A recent multi-institutional retrospective analysis of forty-four treatment naïve or relapsed MDS patients (IPSS-R very high risk (41\%), poor or very poor-risk cytogenetics (43\%), therapy-related MDS (34\%), prior treatment with HMA (73\%), and $>10 \%$ marrow blasts (57\%)) treated with a combination of venetoclax with either azacitidine or decitabine, yielded an overall response rate of $59 \%$ with two-thirds of patients successfully bridged to $\mathrm{AHSCT}^{41}$. Response breakdown was as follows: $14 \% \mathrm{CR}, 27 \% \mathrm{mCR}$ with hematologic improvement, and $18 \% \mathrm{mCR}$ without hematological improvement. The vast majority of patients (79\%) received either 400 or $200 \mathrm{mg}$ venetoclax which was administered for 28 days in $77 \%$ of cases. Of note, onefifth of patients discontinued therapy due to myelosuppression. Patients with very poor-risk IPSS-R cytogenetic abnormalities were less likely to respond with an overall response of $30 \%$ whereas prior HMA exposure did not impact response.

Two additional retrospective studies by Azizi et al $(n=20)$ and Cortes et al. $(n=12)$ that included both treatment naïve MDS and those with HMA failure reported CR plus $\mathrm{mCR}$ rate of $65 \%$ and $33 \%$. respectively ${ }^{42,43}$. In the latter study which was inclusive of three patients with CMML and ten patients with HMA failure, venetoclax was administered in combination with HMA in two-thirds of the patients with the remainder receiving various combinations of venetoclax with low-dose cytarabine, cladribine, mylotarg, CPX-351, or ruxolinitib.

In terms of other chronic myeloid malignancies, limited data exist on venetoclax use in MPN and MDS/MPN overlap syndromes such as CMML. In myelofibrosis, clinical efforts are currently focused on a related drug, navitoclax, in combination with ruxolitinib ${ }^{44}$. We recently reported outcomes with venetoclax and HMA combination therapy in 12 patients with blast-phase MPN, with an overall response rate of $42 \%$, comprising of three patients achieving CR (25\%) and two with partial response (PR). Impressively, three of five responding patients transitioned to $\mathrm{AHSCT}^{45}$. In comparison to historical controls from the Mayo Clinic database of patients with blastphase-MPN treated with HMA alone $(n=26)$ or intensive chemotherapy $(n=69), \mathrm{CR}$ rate of $25 \%$ with HMA plus venetoclax was higher compared to those receiving HMA alone $(4 \% ; p=0.048)$ and both were inferior to those receiving intensive chemotherapy $(35 \% ; p<0.0001)$; furthermore, an additional $24 \%$ of patients receiving intensive chemotherapy achieved CRi but none among patients receiving HMA alone or HMA with venetoclax ${ }^{46}$.

\section{Practice points with venetoclax use}

Venetoclax at $400 \mathrm{mg}$ orally daily is administered most frequently in combination with hypomethylating agents (azacitidine $75 \mathrm{mg} / \mathrm{m}^{2}$ subcutaneously or intravenous $\times$ 7 days or decitabine $20 \mathrm{mg} / \mathrm{m}^{2}$ intravenously days $1-5$ every 28 days). The combination of venetoclax $600 \mathrm{mg}$ orally daily with low-dose cytarabine subcutaneously at $20 \mathrm{mg} / \mathrm{m}^{2}$ SC days $1-10$ every 28 days is considered in patients with prior HMA exposure. On the other hand, venetoclax monotherapy is rarely used due to its limited 
activity. Concomitant use of medications that inhibit CYP3A4 and P-glycoprotein, particularly azole antifungals (i.e. fluconazole, voriconazole, and posaconazole) mandate venetoclax dose adjustments ${ }^{47}$. In other words, venetoclax dose with fluconazole is $200 \mathrm{mg}$ (half dose) and with voriconazole or posaconazole is $100 \mathrm{mg}$ daily (quarter dose). At our institution, we routinely prescribe antimicrobial prophylaxis for AML patients with acyclovir and levofloxacin in addition to azole antifungal prophylaxis $^{48}$. Fluconazole is preferred in treatment naïve elderly patients while posaconazole is used in treatment naïve young AML patients or in the relapsed setting. Duration of venetoclax therapy is typically 28 days for cycle 1 in AML vs 14 days for MDS.

Newly diagnosed AML patients are hospitalized for venetoclax dose ramp up over 3 days $(100,200$, and $400 \mathrm{mg}$ without azoles, 50, 100, and 200 with fluconazole, or 20,50 , and $100 \mathrm{mg}$ with posaconazole). Tumor lysis prophylaxis with saline hydration and allopurinol is instituted upon admission. We consider febuxostat in instances of renal dysfunction ${ }^{49}$ while rasburicase $6 \mathrm{mg}$ IV fixed dose is reserved for high-risk patients presenting with hyperleukocytosis and markedly elevated uric $\operatorname{acid}^{50}$. In patients presenting with leukocytosis $>25 \times 10^{9} / \mathrm{L}$, cytoreduction with hydroxyurea is promptly initiated with consideration of leukapharesis if symptomatic hyperleukostasis. We administer venetoclax once leukocyte count is below $25 \times 10^{9} / \mathrm{L}$. As a result, tumor lysis is extremely infrequent in our practice.

Following venetoclax ramp up and completion of HMA therapy, if medically stable, patients are discharged for daily outpatient monitoring with supportive care at our hospital based outpatient unit. The routine use of growth factor support is not encouraged at our institution; however, it is reasonable to consider in case of a non-resolving infection, or prolonged myelosuppression, if MLFS or CRi is achieved. A bone marrow biopsy is performed for response assessment after completion of cycle 1 (day 28) regardless of peripheral blood counts ${ }^{51}$. Once CR is achieved after cycle 1 , we proceed with cycle 2 of therapy without dose modifications if platelets $>100 \times 10^{9} / \mathrm{L}$ and ANC $>1.0 \times 10^{9} / \mathrm{L}$. In the event of CRi post cycle 1 , we interrupt therapy for approximately 2 weeks to allow hematological recovery. If the latter is achieved within the 2-week time frame, we proceed with cycle 2 without dose modifications. If myelosuppression persists beyond two weeks, we interrupt therapy until platelets $>50 \times 10^{9} / \mathrm{L}$ and ANC $>0.5 \times 10^{9} / \mathrm{L}$ with venetoclax administered for 21 days instead of 28 days during cycle 2 . MRD assessment is also recommended once CR/CRi is achieved.

In the situation of persistent/residual AML post cycle 1, we proceed with cycle 2 of therapy noting that median time to first and best response is 1.2 and 2.1 months, respectively. If leukemia persists, without disease progression post cycle 2 , it is reasonable to proceed with cycle 3 if no alternative therapeutic options. In our experience, one-third of patients experienced a delayed response after $\geq 3$ cycles of therapy ${ }^{20}$.

Prolonged myelosuppression and treatment interruptions with subsequent cycles are a frequent occurrence ${ }^{20}$. Once morphological remission is achieved, we recommend delaying next cycle of therapy until platelets $>50 \times 10^{9} / \mathrm{L}$ and ANC $>0.5 \times 10^{9} / \mathrm{L}$. Step wise dose modifications are recommended with each cycle delay such as venetoclax administration for 21 days, followed by 14 and 7 days along with potential dose modifications of HMA; azacitidine $75 \mathrm{mg} / \mathrm{m}^{2}$ for 5 days or decitabine $20 \mathrm{mg} / \mathrm{m}^{2}$ for 3 days.

In frail elderly patients, we recommend a shortened course of venetoclax for 14 days starting cycle 1 . Once $\mathrm{CR} / \mathrm{CRi} / \mathrm{MLFS}$ is achieved in the above instance, treatment cycles may be administered every $6-8$ weeks with low-dose HMA along with an abbreviated course of venetoclax for 7 days.

\section{Discussion}

Over the last 2 years, the approval of venetoclax-based therapy for upfront use in elderly or unfit AML patients has led to a paradigm shift in our management approach for AML, especially with recently reported phase- 3 studies (VIALE-A, and VIALE-C) demonstrating a clear survival benefit with venetoclax combination therapies in treatment-naïve AML patients ${ }^{15,18}$. Remarkably, venetoclaxbased therapy due to its minimal toxicity and high efficacy has enabled a subset of elderly AML patients to proceed to AHSCT. In regard to post-transplant outcomes following ventoclax-based therapy, a recent study highlights favorable transplant outcomes in 32 AML patients, of which 22 patients were transplanted in CR/CRi with 1 year overall survival of $77 \%$ and non-relapse mortality of $9.1 \%^{52}$. With respect to MDS, we await mature trial results documenting superior efficacy with venetoclax and it remains to be seen whether its addition to HMA confers a meaningful survival advantage.

An area of uncertainty with venetoclax-based therapy in AML involves the routine use and choice of antifungal prophylaxis. In clinical trials, azole antifungal prophylaxis was prohibited due to CYP3A4 inhibition since venetoclax is predominantly metabolized by CYP3A4. It remains unclear whether a lower dose of venetoclax administered with azoles compromises response outcomes. Fortunately, in study patients treated with venetoclax plus azacitidine, the incidence of grade $3 / 4$ fungal infections was low at $8 \%$ which was attributed to use of echinocandins in $46 \%$ of patients $^{13}$. In a large retrospective series of 119 AML patients treated at City of Hope with venetoclax plus HMA, $38 \%$ of patients received micafungin, $41 \%$ azole, and $21 \%$ no antifungal prophylaxis, with a low incidence of fungal infection at $5 \%$ in treatment naïve, vs $19 \%$ in 
relapsed AML, and 6\% in responders vs $22 \%$ in nonresponders ${ }^{33}$. In our practice, we prescribe azole antifungal prophylaxis, with posaconazole in relapsed or treatment naïve young AML patients while fluconazole is preferred in elderly patients.

Another unsettled issue is regarding the optimal timing and lack of uniformity of response assessment including MRD assessment ${ }^{51,53}$. Significant variations exist within terminology used to characterize responses other than CR which is defined as $<5 \%$ marrow blasts, platelets $>100 \times$ $10^{9} / \mathrm{L}$ and ANC $>1.0 \times 10^{9} / \mathrm{L}$. On the contrary, the distinction between CRi $(<5 \%$ marrow blasts, and either platelets $>100 \times 10^{9} / \mathrm{L}$ or $\mathrm{ANC}>1.0 \times 10^{9} / \mathrm{L}$ ), CR with incomplete platelet recovery, $(\mathrm{CRp}), \mathrm{CR}$ with partial hematologic recovery $(\mathrm{CRh}) \quad(<5 \%$ blasts in the bone marrow, and partial recovery of peripheral blood counts (platelets $>50 \times 10^{9} / \mathrm{L}$ and ANC $>0.5 \times 10^{9} / \mathrm{L}$ ), and MLFS, (marrow blast $<5 \%$ with $>200$ cells or marrow cellularity $\geq 10 \%$, and no hematological recovery) is not well-defined. The time frame at which response assessment is performed is also variable based on goals of therapy with some physicians obtaining a bone marrow biopsy after completion of a minimum of two cycles of therapy. Similarly, MRD assessment is inconsistent in terms of timing and platform used; multicolor flow cytometry (MFC), digital droplet polymerase chain reaction (PCR), or emerging next generation sequencing technologies ${ }^{54}$. At our institution, multicolor flow cytometry based MRD assessment ${ }^{55}$ is routinely performed through the University of Washington, Seattle, for patients achieving CR/ CRi particularly if AHSCT is being considered. The overall sensitivity of the assay is conservatively estimated as $0.1 \%$. In addition, we have developed an in-house quantitative PCR assay for monitoring type $\mathrm{A}, \mathrm{B}$, and $\mathrm{D}$ NPM1 mutation types with forthcoming assays for $C B F B$ / MYH11 and RUNX1/RUNX1T1 fusions.

In terms of therapeutic decision-making for elderly or unfit AML patients with known FLT3 or IDH1/2 mutations, we are often faced with the choice of venetoclax-based therapy or targeted therapy with either $I D H$ inhibitors (enasidenib/ivosidenib) or FLT3 inhibitors (midostaurin/ gilteritinib). Preliminary results from an ongoing phase Ib/II study on venetoclax plus ivosidenib with or without azacitidine in IDH1 mutated AML and high-risk MDS appear promising ${ }^{56}$. A total of 19 patients were enrolled of which 17 were AML (9 relapsed, 5 treatment naïve and 3 with secondary AML following MDS with progression on HMA) and 2 patients with high-risk MDS with composite CR rate of $78 \%$ overall and $100 \%$ for treatment-naive patients. In addition, half of patients who achieved CR also were MRD negative. In a similar vein, preclinical studies confirm synergistic activity with BCL-2 and FLT3 inhibition ${ }^{57}$ with a phase $1 \mathrm{~b}$ trial of venetoclax with gilteritinib in relapsed/ refractory AML in progress. Of 15 relapsed/refractory AML patients, 10 of which were FLT3 mutated (6 previously treated with FLT3 inhibitors), half achieved composite CR, and another $40 \%$ achieved MLFS ${ }^{58}$. Furthermore, a recently published Phase II study confirms the safety and efficacy of combination therapy with addition of FLT3 inhibitors to decitabine and venetoclax ${ }^{16}$. Together, these findings suggest that future therapies for AML are likely to incorporate targeted agents (IDH/FLT3 inhibitors) into venetoclaxbased regimens.

\section{Author contributions}

N.G. and A.T. reviewed the literature and co-wrote the paper.

Conflict of interest

The authors declare that they have no conflict of interest.

\section{Publisher's note}

Springer Nature remains neutral with regard to jurisdictional claims in published maps and institutional affiliations.

Received: 6 October 2020 Revised: 24 October 2020 Accepted: 5 November 2020

Published online: 23 November 2020

\section{References}

1. Tsujimoto, Y., Finger, L. R., Yunis, J., Nowell, P. C. \& Croce, C. M. Cloning of the chromosome breakpoint of neoplastic B cells with the $t(14 ; 18)$ chromosome translocation. Science. 226, 1097-1099 (1984).

2. Vaux, D. L., Cory, S. \& Adams, J. M. Bcl-2 gene promotes haemopoietic cell survival and cooperates with c-myc to immortalize pre-B cells. Nature. $\mathbf{3 3 5}$ 440-442 (1988).

3. Leverson, J. D. et al. Found in translation: how preclinical research is guiding the clinical development of the BCL2-selective inhibitor Venetoclax. Cancer Discov. 7, 1376-1393 (2017).

4. Andreeff, M. et al. Expression of BCl-2-related genes in normal and AML progenitors: changes induced by chemotherapy and retinoic acid. Leukemia 13, 1881-1892 (1999).

5. Tse, C. et al. ABT-263: a potent and orally bioavailable BCl-2 family inhibitor. Cancer Res. 68, 3421-3428 (2008).

6. Zhang, $\mathrm{H}$. et al. BCl-2 family proteins are essential for platelet survival. Cell Death Differ. 14, 943-951 (2007).

7. Konopleva, M. et al. Mechanisms of apoptosis sensitivity and resistance to the BH3 mimetic ABT-737 in acute myeloid leukemia. Cancer Cell 10, 375-388 (2006).

8. Pan, R. et al. Selective BCL-2 inhibition by ABT-199 causes on-target cell death in acute myeloid leukemia. Cancer Discov. 4, 362-375 (2014).

9. Niu, X. et al. Acute myeloid leukemia cells harboring MLL fusion genes or with the acute promyelocytic leukemia phenotype are sensitive to the BCl-2selective inhibitor ABT-199. Leukemia 28, 1557-1560 (2014).

10. Bogenberger, J. M. et al. BCL-2 family proteins as 5-Azacytidine-sensitizing targets and determinants of response in myeloid malignancies. Leukemia $\mathbf{2 8}$, 1657-1665 (2014).

11. Tsao, T. et al. Concomitant inhibition of DNA methyltransferase and BCL-2 protein function synergistically induce mitochondrial apoptosis in acute myelogenous leukemia cells. Ann. Hematol. 91, 1861-1870 (2012).

12. DiNardo, C. D. et al. Safety and preliminary efficacy of venetoclax with decitabine or azacitidine in elderly patients with previously untreated acute myeloid leukaemia: a non-randomised, open-label, phase $1 \mathrm{~b}$ study. Lancet Oncol. 19, 216-228 (2018).

13. DiNardo, C. D. et al. Venetoclax combined with decitabine or azacitidine in treatment-naive, elderly patients with acute myeloid leukemia. Blood 133, 7-17 (2019).

14. Pollyea, D. A. et al. Venetoclax with azacitidine disrupts energy metabolism and targets leukemia stem cells in patients with acute myeloid leukemia. Nat. Med. 24, 1859-1866 (2018). 
15. DiNardo, C. D. et al. Azacitidine and venetoclax in previously untreated acute myeloid leukemia. N. Engl. J. Med. 383, 617-629 (2020).

16. DiNardo, C. D. et al. 10-day decitabine with venetoclax for newly diagnosed intensive chemotherapy ineligible, and relapsed or refractory acute myeloid leukaemia: a single-centre, phase 2 trial. Lancet Haematol. 7, e724-e736 (2020).

17. Wei, A. H. et al. Venetoclax combined with low-dose cytarabine for previously untreated patients with acute myeloid leukemia: results from a Phase Ib/II study. J. Clin. Oncol. 37, 1277-1284 (2019).

18. Wei, A. H. et al. Venetoclax plus LDAC for newly diagnosed AML ineligible for intensive chemotherapy: a phase 3 randomized placebo-controlled trial. Blood 135, 2137-2145 (2020).

19. Winters, A. C. et al. Real-world experience of venetoclax with azacitidine for untreated patients with acute myeloid leukemia. Blood Adv. 3, 2911-2919 (2019).

20. Morsia, E. et al. Venetoclax and hypomethylating agents in acute myeloid leukemia: Mayo Clinic series on 86 patients. Am. J. Hematol. 95, 1511-1521 (2020).

21. Nanah, R. et al. Outcome of elderly patients after failure to hypomethylating agents given as frontline therapy for acute myeloid leukemia: single institution experience. Am. J. Hematol. 92, 866-871 (2017).

22. Konopleva, M. et al. Efficacy and biological correlates of response in a phase II study of venetoclax monotherapy in patients with acute myelogenous leukemia. Cancer Discov. 6, 1106-1117 (2016).

23. Chyla, B. et al. Genetic biomarkers of sensitivity and resistance to venetoclax monotherapy in patients with relapsed acute myeloid leukemia. Am. J. Hematol. 93, E202-E205 (2018).

24. DiNardo, C. D. et al. Molecular patterns of response and treatment failure after frontline venetoclax combinations in older patients with AML. Blood 135 791-803 (2020).

25. DiNardo, C. D. et al. Clinical experience with the BCL2-inhibitor venetoclax in combination therapy for relapsed and refractory acute myeloid leukemia and related myeloid malignancies. Am. J. Hematol. 93, 401-407 (2018).

26. Aldoss, I. et al. Efficacy of the combination of venetoclax and hypomethylating agents in relapsed/refractory acute myeloid leukemia. Haematologica 103, e404-e407 (2018).

27. Ram, R. et al. Venetoclax in patients with acute myeloid leukemia refractory to hypomethylating agents-a multicenter historical prospective study. Ann. Hematol. 98, 1927-1932 (2019).

28. Gaut, D. et al. Venetoclax combination therapy in relapsed/refractory acute myeloid leukemia: a single institution experience. Leuk. Res. 90, 106314 (2020).

29. Wang, Y. W. et al. Cytogenetics and mutations could predict outcome in relapsed and refractory acute myeloid leukemia patients receiving $B C L-2$ inhibitor venetoclax. Ann. Hematol. 99, 501-511 (2020).

30. Huemer, F. et al. Durable remissions with venetoclax monotherapy in secondary AML refractory to hypomethylating agents and high expression of BCL-2 and/or BIM. Eur. J. Haematol. 102, 437-441 (2019).

31. Ganzel, C. et al. Venetoclax is safe and efficacious in relapsed/refractory AML. Leuk. Lymphoma 61, 2221-2225 (2020).

32. Byrne, $M$. et al. The use of venetoclax-based salvage therapy for posthematopoietic cell transplantation relapse of acute myeloid leukemia. Am. J. Hematol. 85, 1006-1014 (2020).

33. Aldoss, l. et al. Invasive fungal infections in acute myeloid leukemia treated with venetoclax and hypomethylating agents. Blood Adv. 3, 4043-4049 (2019).

34. Aldoss, l. et al. Association of leukemia genetics with response to venetoclax and hypomethylating agents in relapsed/refractory acute myeloid leukemia. Am. J. Hematol. 94, E253-E255 (2019).

35. Bewersdorf, J. P. et al. Venetoclax as monotherapy and in combination with hypomethylating agents or low dose cytarabine in relapsed and treatment refractory acute myeloid leukemia: a systematic review and meta-analysis. Haematologica 105, https://doi.org/10.3324/haematol.2019.242826 (2020).

36. Aldoss, I. et al. Venetoclax and hypomethylating agents in TP53-mutated acute myeloid leukaemia. Br. J. Haematol. 187, e45-e48 (2019).

37. Wei, A. H. et al. A phase 1b study evaluating the safety and efficacy of venetoclax in combination with azacitidine in treatment-naive patients with higher-risk myelodysplastic syndrome. Blood 134, 568 (2019).
38. Garcia, J. S. et al. The prognostic impact of cytogenetic scors in patients with higher-risk myelodysplastic syndrome treated with venetoclax and azacitidine in a phase 1 study. EHA Abstr. 294712 (2020).

39. Zeidan, A. M. et al. A phase $1 \mathrm{~b}$ study evaluating the safety and efficacy of venetoclax as monotherapy or in combination with azacitidine for the treatment of relapsed/refractory myelodysplastic syndrome. Blood 134, 565 (2019).

40. Zeida, A. M. et al. A Phase 1B study evaluating the safety and efficacy of venetoclax combination with azacitidine for the treatment of relapsed/ refractory myelodysplastic syndrome. EHA Abstr. 295008 (2020).

41. Ball, B. J. et al. Venetoclax and hypomethylating agents (HMAs) induce high response rates in MDS, including patients after HMA therapy failure. Blood Adv. 4, 2866-2870 (2020)

42. Azizi, A. et al. Venetoclax and hypomethylating agent therapy in high risk myelodysplastic syndromes: a retrospective evaluation of a real-world experience. Leuk. Lymphoma 61, 2700-2707 (2020).

43. Cortes, J. et al. Activity of venetoclax-based therapy in myelodysplastic syndrome (MDS). Blood 134, 1726 (2019).

44. Harrison, C. N. et al. Results from a phase 2 study of navitoclax in combination with ruxolitinib in patients with primary or secondary myelofibrosis. Blood 134, 671 (2019).

45. Gangat, N. et al. Venetoclax plus hypomethylating agent in blast phase myeloproliferative neoplasm: preliminary experience with 12 patients. Br. J. Haematol. https://doi.org/10.1111/bjh.17084 (2020) (In press).

46. Tefferi, A. et al. Blast phase myeloproliferative neoplasm: Mayo-AGIMM study of 410 patients from two separate cohorts. Leukemia 32, 1200-1210 (2018).

47. Agarwal, S. K. et al. Management of venetoclax-posaconazole interaction in acute myeloid leukemia patients: evaluation of dose adjustments. Clin. Ther. 39, 359-367 (2017).

48. Taplitz, R. A. et al. Antimicrobial prophylaxis for adult patients with cancerrelated immunosuppression: ASCO and IDSA clinical practice guideline update. J. Clin. Oncol. 36, 3043-3054 (2018).

49. Tamura, K. et al. Efficacy and safety of febuxostat for prevention of tumor lysis syndrome in patients with malignant tumors receiving chemotherapy: a phase III, randomized, multi-center trial comparing febuxostat and allopurinol. Int. J. Clin. Oncol. 21, 996-1003 (2016).

50. Vines, A. N., Shanholtz, C. B. \& Thompson, J. L. Fixed-dose rasburicase 6 mg for hyperuricemia and tumor lysis syndrome in high-risk cancer patients. Ann. Pharmacother. 44, 1529-1537 (2010).

51. Dohner, $\mathrm{H}$. et al. Diagnosis and management of AML in adults: 2017 ELN recommendations from an international expert panel. Blood 129, 424-447 (2017).

52. Sandhu, K. S. et al. Outcome of allogeneic hematopoietic cell transplantation after venetoclax and hypomethylating agent therapy for acute myeloid leukemia. Biol. Blood Marrow Transplant https://doi.org/10.1016/j. bbmt.2020.08.027 (2020) (In press).

53. Herold, T. et al. Validation and refinement of the revised 2017 European LeukemiaNet genetic risk stratification of acute myeloid leukemia. Leukemia https://doi.org/10.1038/s41375-020-0806-0 (2020).

54. Schuurhuis, G. J. et al. Minimal/measurable residual disease in AML: a consensus document from the European LeukemiaNet MRD Working Party. Blood 131, 1275-1291 (2018).

55. Al-Mawali, A., Gillis, D. \& Lewis, I. The role of multiparameter flow cytometry for detection of minimal residual disease in acute myeloid leukemia. Am J Clin Pathol 131, 16-26 (2009).

56. Lachowiez, C. A. et al. Phase lb/II study of the IDH1-mutant inhibitor ivosidenib with the BCL2 inhibitor venetoclax +/- azacitidine in IDH1-mutated hematologic malignancies. J. Clin. Oncol. 38, 7500 (2020).

57. Mali, R. S. et al. Venetoclax combines synergistically with FLT3 inhibition to effectively target leukemic cells in FLT3-ITD+ acute myeloid leukemia models. Haematologica https://doi.org/10.3324/haematol.2019.244020 (2020).

58. Perl, A. E. et al. Venetoclax in combination with gilteritinib in patients with relapsed/refractory acute myeloid leukemia: a phase 1b study. Blood 134, 3910 (2019).

59. Joshi, M. et al. Salvage use of venetoclax-based therapy for relapsed AML post allogeneichematopoietic cell transplantation. BCJ (2020) (In press). 